\title{
Quinazoline analog HMJ-30 inhibits angiogenesis: Involvement of endothelial cell apoptosis through ROS-JNK-mediated death receptor 5 signaling
}

\author{
CHI-CHENG LU ${ }^{1}$, HAO-PING CHEN ${ }^{2}$, JO-HUA CHIANG ${ }^{3}$, YI-AN JIN ${ }^{4}$, SHENG-CHU KUO ${ }^{5}$, \\ TIAN-SHUNG WU ${ }^{3}$, MANN-JEN HOUR ${ }^{6}$, JAI-SING YANG ${ }^{7}$ and YU-JEN CHIU ${ }^{8}$
}

\author{
${ }^{1}$ Department of Food Science and Biotechnology, National Chung Hsing University, Taichung 402; \\ ${ }^{2}$ Department of Biochemistry, Tzu Chi University, Hualien 970; ${ }^{3}$ Department of Chemistry, National Cheng Kung University, \\ Tainan $701 ;{ }^{4}$ Department of Dermatology, Taipei Medical University Hospital, Taipei $110 ;{ }^{5}$ Graduate Institute \\ of Pharmaceutical Chemistry, ${ }^{6}$ School of Pharmacy and ${ }^{7}$ Department of Pharmacology, School of Medicine, \\ China Medical University, Taichung 404; ${ }^{8}$ Division of Reconstructive and Plastic Surgery, \\ Department of Surgery, Taipei Veterans General Hospital, Taipei 112, Taiwan, R.O.C.
}

Received March 26, 2014; Accepted May 14, 2014

DOI: $10.3892 / o r .2014 .3250$

\begin{abstract}
The aim of the present study was to explore the effect of 6-fluoro-2-(3-fluorophenyl)-4-(cyanoanilino) quinazoline (HMJ-30) on the anti-angiogenic properties and apoptosisrelated mechanism of human umbilical vein endothelial cells (HUVECs). In this study, HMJ-30 dose- and time-dependently inhibited the viability of HUVECs. We also found that HMJ-30 enhanced disruption of tube-like structures and suppressed cell migration in HUVECs after vascular endothelial growth factor (VEGF) induction. HMJ-30 was also observed to inhibit vessel branching and sprouting in chicken chorioallantoic membrane (CAM). Microsprouting induced by VEGF in the rat aortic ring and blood vessel formation in a mouse Matrigel plug were individually suppressed by HMJ-30. In an in vitro study, HMJ-30 induced the apoptotic death of HUVECs as indicated by DNA fragmentation and promoted reactive oxygen species (ROS) production as determined by flow cytometric assay. In addition, extrinsic caspase signaling (caspase- 8 and -3) was activated in the HMJ-30-treated HUVECs and their inhibitors were applied to assess the signal transduction. We investigated the upstream of the death receptor pathway and further observed that the levels of death receptor 5 (DR5) and phosphorylated c-Jun N-terminal kinase (JNK) signals were upregulated in HUVECs following HMJ-30 challenge,
\end{abstract}

Correspondence to: Dr Jai-Sing Yang, Department of Pharmacology, School of Medicine, China Medical University, No. 91 Hsueh-Shih Road, Taichung 40402, Taiwan, R.O.C.

E-mail: jaisingyang@gmail.com

Dr Yu-Jen Chiu, Division of Reconstructive and Plastic Surgery, Department of Surgery, Taipei Veterans General Hospital, No. 201 Sec. 2, Shipai Road, Taipei 112, Taiwan, R.O.C.

E-mail: chiou70202@yahoo.com.tw

Key words: HMJ-30, angiogenesis, HUVECs, apoptosis, ROS, MAPK/JNK signal which was confirmed by a JNK-specific inhibitor (SP600125). Hence, HMJ-30-induced endothelial cell apoptosis involved the ROS/JNK-regulated DR5 pathway. In summary, HMJ-30 may provide a potential therapeutic effect for the anti-vascular targeting of angiogenesis during cancer treatment.

\section{Introduction}

Angiogenesis is a vital and complicated process involving endothelial cells, pericytes and the environment, which is executed to ensure normal physiological responses, including wound healing, embryonic development and bone remodeling $(1,2)$. On the contrary, it also plays a critical role in the pathogenesis of multiple diseases and cancer progression (3). Tumor-induced angiogenesis not only provides the essential blood supply but also allows cancer cell metastasis by allowing cells to enter the circulation $(4,5)$. Without a blood circulation supplement, a tumor is unable to grow beyond a critical size or to metastasize to another organ. Thus, tumor neovascularization has become a potential therapeutic target. Anti-angiogenetic strategies attempt to destroy existing vessels and inhibit new vessel formation to starve and prison tumor cells $(6,7)$. Angiogenesis inhibitors have now been approved for clinical application, and vascular endothelial growth factor (VEGF) was initially identified as a critical angiogenesis promoter $(5,8)$. Bevacizumab (Avastin; Roche/Genentech) is a specific VEGF antibody used as an angiogenesis inhibitor (9), and this agent has been used with chemotherapy or cytokine therapy for various advanced metastatic cancers (10-12). Furthermore, multiple-targeted pan-VEGF receptor tyrosine kinase inhibitors were subsequently approved for the treatment of advanced cancer and age-related macular degeneration. With treatment, the survival of cancer patients is generally prolonged by several months $(13,14)$.

However, targeting of the VEGF pathway has not proven as efficacious as hoped. Tumor cells are able to evade antiVEGF therapy after a period of treatment paricularly certain 
late-stage cancers $(1,8)$. Multiple plausible mechanisms of escape and resistance have been reported, including upregulation of alternative pathways in selected tumor clones, providing vascular progenitors and modulators with resilient systems to support a neovascular response (15) and tumor-associated endothelial cell genetic instability and resistance to anti-VEGF therapy (16). Moreover, induction of endothelial cell apoptosis leads to dysfunction of blood vessel to support non-nutrients during angiogenesis $(6,7)$. As a result, engineering new inhibitors with which to target angiogenesis through pathways other than VEGF signaling is increasingly important.

Quinazoline derivatives have been found to possess various pharmacological effects, including anti-inflammatory and anticancer activities (17-20). In our laboratory, we synthesized a series of quinazoline compounds with fluorine as human anticancer candidates (21). One of these compounds, 6-fluoro-2-(3-fluorophenyl)-4-(cyanoanilino) quinazoline (HMJ-30), has been reported to induce apoptotic death through induction of oxidative stress and upregulation of ataxia telangiectasia mutated (ATM)/p53 signaling in U-2 OS human osteosarcoma cells (20). Additionally, xenograft tumor growth of osteosarcoma in nude mice was inhibited by HMJ-30 (unpublished data). Since angiogenesis contributes to a poor prognosis in human osteosarcoma (23), the study of the anti-angiogenic mechanism of osteosarcoma cells may lead to the development of novel and successful strategies for the treatment of osteosarcoma. The inhibitory effects on the angiogenic response by HMJ-30 and the molecular mechanisms of its cytotoxic effects on HUVECs remain unclear; thus these issues were investigated in the present study. We focused on the vascular targeting effects and resulted in apoptosis of endothelial cells triggered by HMJ-30.

\section{Materials and methods}

Chemicals and reagents. HMJ-30 was synthesized by Dr Mann-Jen Hour, and its chemical structure is shown in Fig. 1A. Caspase-3, caspase- 8 and caspase- 9 colorimetric assay kits, caspase-3 inhibitor Z-DEVD-FMK, caspase-8 inhibitor Z-IETD-FMK and recombinant human VEGF were purchased from R\&D Systems Inc. (Minneapolis, MN, USA). Materials and chemicals for electrophoresis were obtained from Bio-Rad Laboratories, Inc. (Hercules, CA, USA). Primary antibodies (Fas/CD95, DR4, DR5, TNFR and actin) and horseradish peroxidase (HRP)-conjugated secondary antibodies against rabbit or mouse immunoglobulin were obtained from Santa Cruz Biotechnology (Santa Cruz, CA, USA). The other antibodies for immunoblotting were purchased from Cell Signaling Technology (Beverly, MA, USA). All other chemicals were of analytical grade and were obtained from Sigma-Aldrich Corp. (St. Louis, MO, USA) unless otherwise stated.

Cell culture.Human umbilical vein endothelial cells (HUVECs, $\mathrm{CD} 1^{+}>99 \%$ ) were purchased from the Bioresource Collection and Research Center (BCRC, Hsinchu, Taiwan) and maintained in Medium 200 (Gibco Life Technologies, Carlsbad, CA, USA) supplemented with low serum growth supplement (LSGS; Gibco Life Technologies) at $37^{\circ} \mathrm{C}$ in a humidified atmosphere with $5 \% \mathrm{CO}_{2}$. HUVECs were used between the second and the fourth generation.
Cell viability. HUVECs in 96-well plates at a density of $5 \times 10^{3}$ cells/well were exposed to HMJ-30 at various concentrations $(5,10,20$ and $30 \mu \mathrm{M})$ for 24 and $48 \mathrm{~h}$. The effects of HMJ-30-induced cytotoxicity were measured using the 3-(4,5-dimethylthiazol-2-yl)-2,5-diphenyltetrazolium bromide (MTT) assay following previously reported methods $(24,25)$.

Endothelial tube formation assay. HUVECs ( $1 \times 10^{5}$ cells/well) were seeded on a 48-well culture plate precoated with Matrigel (BD Biosciences, Bedford, MA, USA) containing $20 \mathrm{ng} / \mathrm{ml}$ VEGF as previously described (26) and were subsequently treated with or without different concentrations of HMJ-30 (5, 10 and $20 \mu \mathrm{M})$. At $16 \mathrm{~h}$ post-seeding, cells were fixed with $4 \%$ paraformaldehyde prior to capturing images under an inverted phase-contrast microscope.

Endothelial cell migration assay. HUVECs $\left(5 \times 10^{4}\right.$ cells $\left./ \mathrm{ml}\right)$ were initially incubated in a Transwell (Millicell Cell Culture Insert, polycarbonate, $8.0 \mu \mathrm{m}$; EMD Millipore Corporation, Billerica, MA, USA) for $4 \mathrm{~h}$. Subsequently, different concentrations of HMJ-30 (5, 10 and $20 \mu \mathrm{M})$ were added into individual wells and the procedure was followed as described in the protocols by Chiang et al (27). The migrating cells were quantified by counting the cell number after being stained in three random fields/well with a microscope.

Chick embryo assay. In vivo angiogenesis assays have made important progress in elucidating the mechanism of action of angiogenesis-influencing factors (28). After 7-9 days, the chick chorioallantoic membrane (CAM) in embryos was exposed to final concentrations of 10 and $20 \mu \mathrm{M}$ of HMJ-30 by making a window in the egg shell. The window was sealed and eggs were re-incubated for an appropriate incubation period.

Ex vitro rat aortic ring assay. The aortic ring explant cultures were carried out as previously described by Pyun et al with various modification (29). Six-week old male Sprague-Dawley (SD) rats were obtained from BioLASCO Taiwan Co., Ltd. (Taipei, Taiwan) and subsequently the aortic rings were collected to test vessel sprouting as previously described $(27,30)$.

Matrigel plug assay. Matrigel basement membrane matrix (BD Biosciences) containing $200 \mathrm{ng} / \mathrm{ml}$ VEGF was injected subcutaneously into the dorsal region of 6- to 8-week old BALB/c nude mice obtained from the National Laboratory Animal Center (Taipei, Taiwan). After Matrigel plug solidification according to a previous method $(27,31), \mathrm{HMJ}-30$ at 10 and $20 \mathrm{mg} / \mathrm{kg}$ was administered intraperitoneally daily before mice were sacrificed. On day 7, Matrigel pellets were harvested and processed for staining and then photographed. Neovascularization was quantified by measuring the hemoglobin of the plugs using the Drabkin method (Drabkin's reagent; Sigma-Aldrich Corp.).

Detection of HUVEC morphology and DNA content analysis by flow cytometry. Following HMJ-30 treatment $(5,10$ or $20 \mu \mathrm{M})$, cells in 24-well plates at a density of $1 \times 10^{5}$ cells/ well for $24 \mathrm{~h}$ with VEGF stimulation were harvested before morphological changes were examined by a phase-contrast 
A<smiles>N#Cc1ccc(Nc2nc(-c3cccc(F)c3)nc3ccc(F)cc23)cc1</smiles>

HMJ-30

C

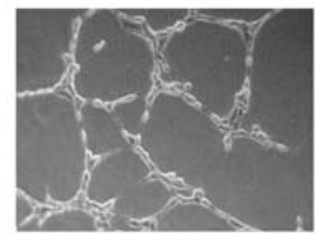

CTL

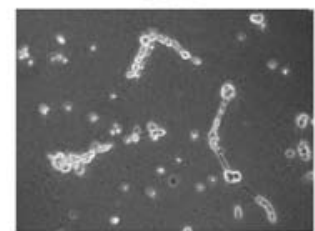

HMJ-30 [10 $\mu \mathrm{M}]$

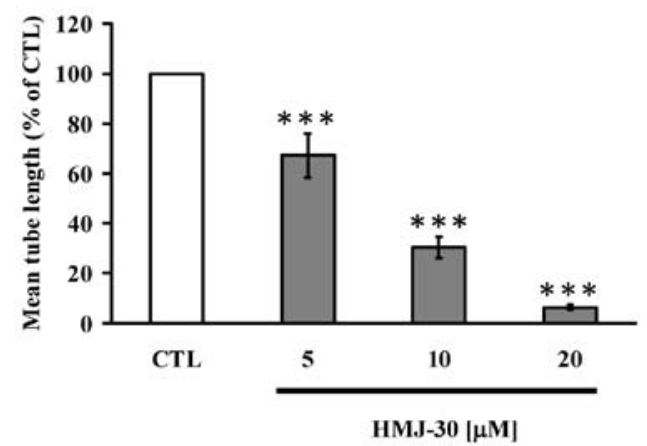

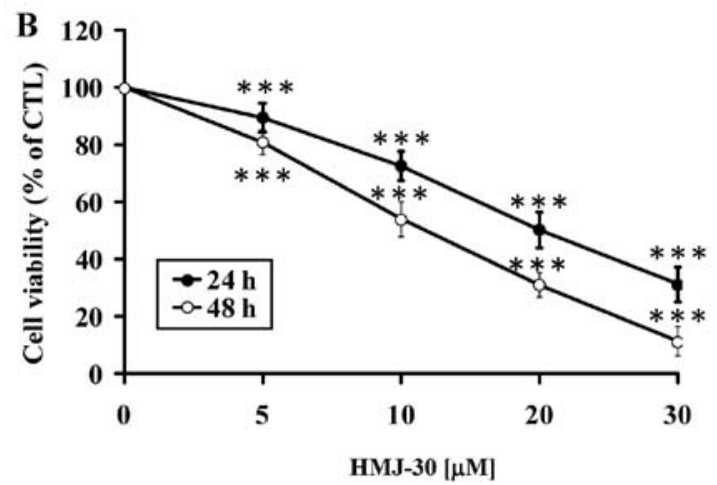

D

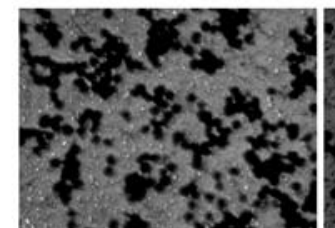

CTL

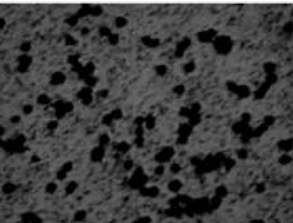

HMJ-30 [5 $\mu \mathrm{M}]$

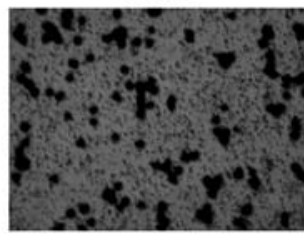

HMJ-30 [10 $\mu \mathrm{M}]$

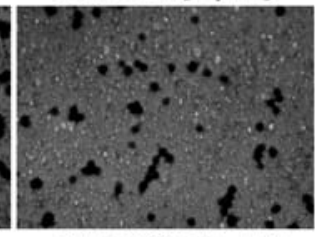

HMJ-30 [20 $\mu \mathrm{M}]$

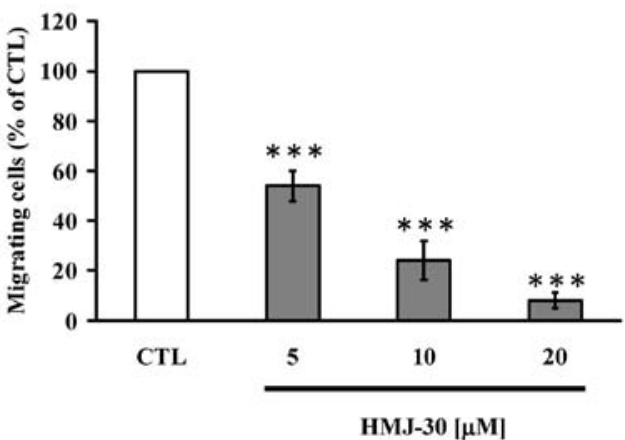

Figure 1. Effect of HMJ-30 on tube formation and cell migration of HUVECs in vitro. (A) Molecular structure of HMJ-30. (A) HUVECs were treated without or with HMJ-30 $(5,10,20$ or $30 \mu \mathrm{M})$ for 24 or $48 \mathrm{~h}$, or $0.1 \%$ DMSO as a vehicle control, and MTT assay was used to evaluate cell viability. (C) The tube-like structures stimulated by VEGF in HUVECs were assessed after treatment with or without HMJ-30 and images were captured with an inverted microscope before tube length was quantified. (D) Cell migratory ability after VEGF induction was detected by Transwell migration assay as mentioned in Materials and methods. Each result is expressed as the mean $\pm \operatorname{SEM}(n=3) .{ }^{* * * *} \mathrm{p}<0.001$ significant difference compared with the untreated control (CTL).

microscope. The collected cells were fixed in $75 \%$ ethanol overnight at $-20^{\circ} \mathrm{C}$ before being stained with $0.1 \mathrm{M}$ phosphate/ citric acid buffer $\left(0.2 \mathrm{M} \mathrm{NaHPO}_{4}, 0.1 \mathrm{M}\right.$ citric acid, $\left.\mathrm{pH} 7.8\right)$ and $40 \mu \mathrm{g} / \mathrm{ml}$ propidium iodide for $30 \mathrm{~min}$ at room temperature in darkness. The stained cells were determined with FACSCalibur (BD Biosciences, Franklin Lakes, NJ, USA).

Apoptotic assay. Following treatment with 5, 10 and $20 \mu \mathrm{M}$ of HMJ-30 for $24 \mathrm{~h}$ or without treatment, apoptosis in the HUVECs was determined by terminal deoxynucleotidyl transferase dUTP nick end labeling (TUNEL) assay, using an In Situ Cell Death Detection kit, fluorescein (Roche Diagnostics $\mathrm{GmbH}$, Mannheim, Germany) and flow cytometric analysis. Following HMJ-30 exposure, cells were prepared for detect- tion of DNA fragmentation following a previously reported method $(32,33)$.

Measurements of ROS production and cell viability following pretreatment with $N$-acetylcysteine (NAC) or catalase. HUVECs were cultured with $20 \mu \mathrm{M}$ HMJ-30 for $0,3,6$ and $12 \mathrm{~h}$. Cells were then harvested and labeled with $20 \mu \mathrm{M}$ 2,7-dichlorodihydrofluorescein diacetate $\left(\mathrm{H}_{2}\right.$ DCF-DA) (a specific ROS fluorescence probe) at $37^{\circ} \mathrm{C}$ for $30 \mathrm{~min}$. Consequently, ROS production was analyzed for fluorescence intensity by flow cytometry. Cells were pre-incubated with or without $10 \mathrm{mM}$ NAC (an antioxidant) or $5 \mu \mathrm{g} / \mathrm{ml}$ catalase for $1 \mathrm{~h}$ before exposure to $20 \mu \mathrm{M} \mathrm{HMJ}-30$ for $24 \mathrm{~h}$. Cell viability was determined by MTT assay as described above. 


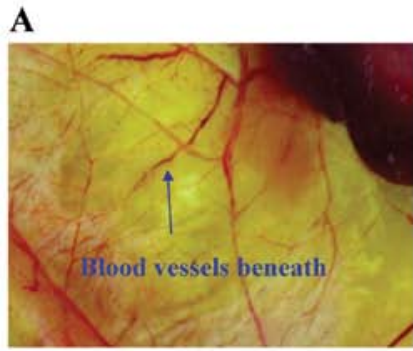

CTL

B

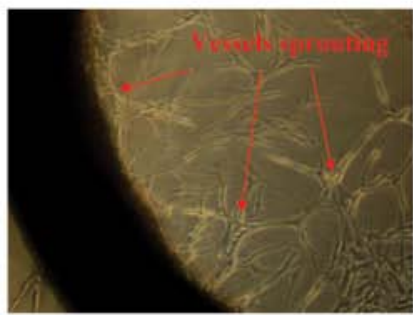

CTL

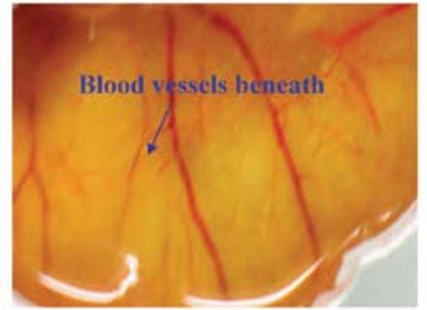

HMJ-30 [10 $\mu \mathrm{M}]$

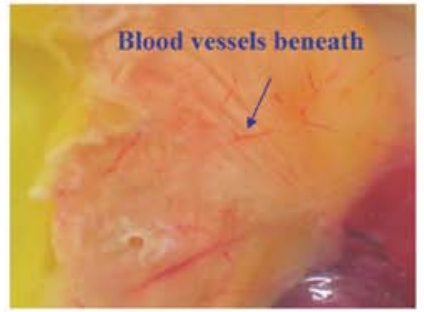

HMJ-30 [20 $\mu \mathrm{M}]$

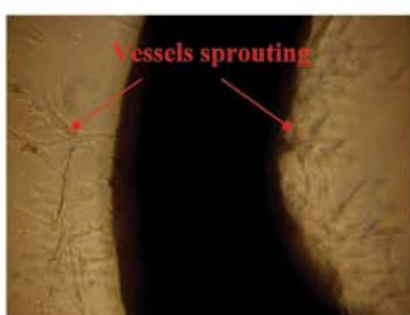

HMJ-30 [10 $\mu \mathrm{M}]$

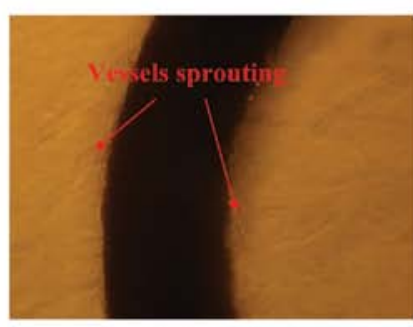

HMJ-30 [20 $\mu \mathrm{M}]$

C

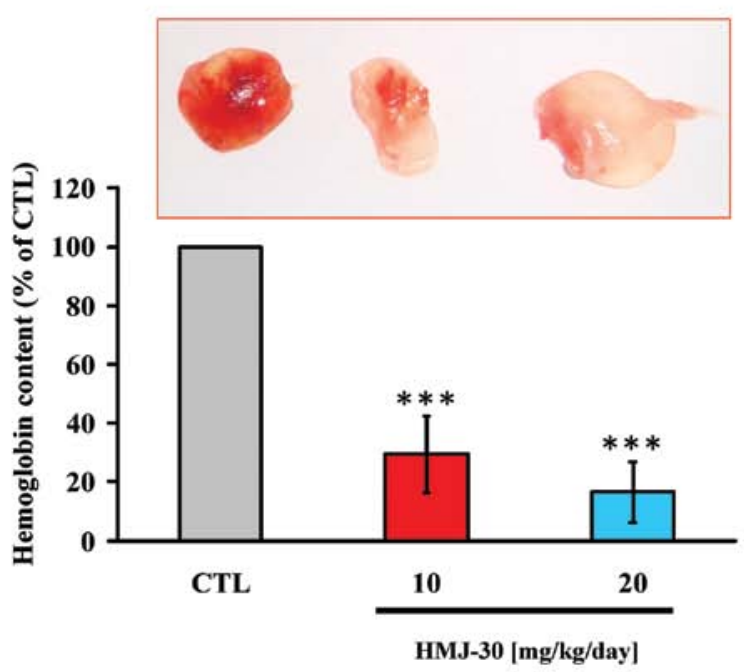

Figure 2. Inhibitory effect of HMJ-30 on the angiogenic responses in vivo and ex vivo. (A) CAM assay to express blood vessels beneath was used for assessing anti-angiogenesis in the presence or absence of HMJ-38 challenge. A total of three randomly selected regions of each sample were visualized and photographed. (B) The aorta rings with tube-like structure formation are shown and respective samples were photographed under a microscope. (C) Representative macroscopic images from the Matrigel plug bioassay to determine the anti-angiogenic effect of HMJ-30 at 10 and $20 \mathrm{mg} / \mathrm{kg}$. The quantification of vessel formation inside the plugs was assayed by the Drabkin method. Data are represented as the mean \pm SEM of at least three animals. ${ }^{* * *}$ p $<0.001$, significantly different in comparison to VEGF alone (CTL).

Determination of caspase-3, caspase-8 and caspase-9 activities and the effect of their specific inhibitors. HUVECs $\left(5 \times 10^{6}\right.$ cells $)$ were incubated in 75 -T flasks and treated with $20 \mu \mathrm{M}$ of HMJ-30 for 0,12 and $24 \mathrm{~h}$. Cells were then harvested to assess the relative caspase activity using caspase-3, caspase- 8 and caspase- 9 colorimetric assay kits (R\&D Systems Inc.) following the manufacturer's instructions. Cells were pretreated with $10 \mu \mathrm{M}$ Z-DEVD-FMK (a specific caspase-3 inhibitor) or $10 \mu \mathrm{M}$ Z-IETD-FMK (a specific caspase-8 inhibitor) for $1 \mathrm{~h}$ and MTT assay was performed as detailed above.

Western blot analysis. HUVECs $\left(5 \times 10^{6}\right.$ cells) were incubated in $20 \mu \mathrm{M} \mathrm{HMJ}-30$ for $0,3,6,12$ or $24 \mathrm{~h}$. Cells were harvested, and an equal amount of protein extract from the cell lysate was separated on $10 \%$ SDS-polyacrylamide electrophoresis gels (SDS-PAGE) as previously described $(27,34)$. The appropriate primary antibodies were hybridized and the specific protein signals were then observed using the Immobilon Western HRP substrate kit (Merck Millipore) after using the HRP-conjugated secondary antibodies. Actin served as an internal control to ensure equal loading, and NIH ImageJ 1.47 software was used to perform the densitometric quantification of each band.

Statistical analysis. Data are represented as mean \pm standard error of the mean (SEM) from at least three separate experiments. Statistical calculations of the data were carried out 

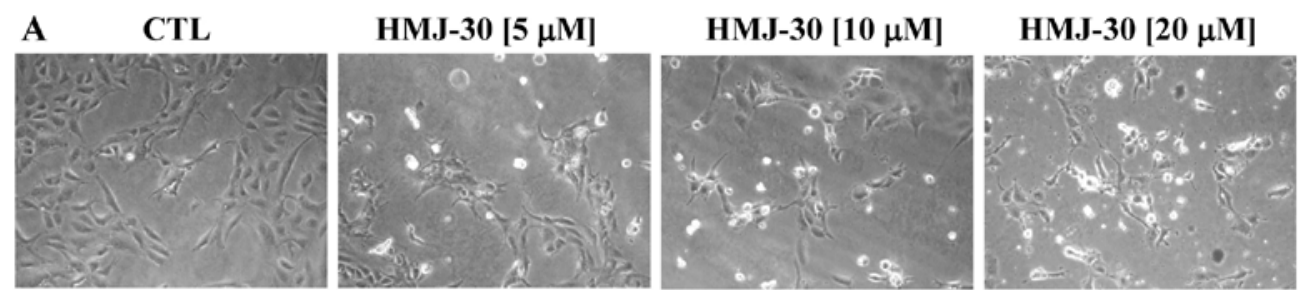

B
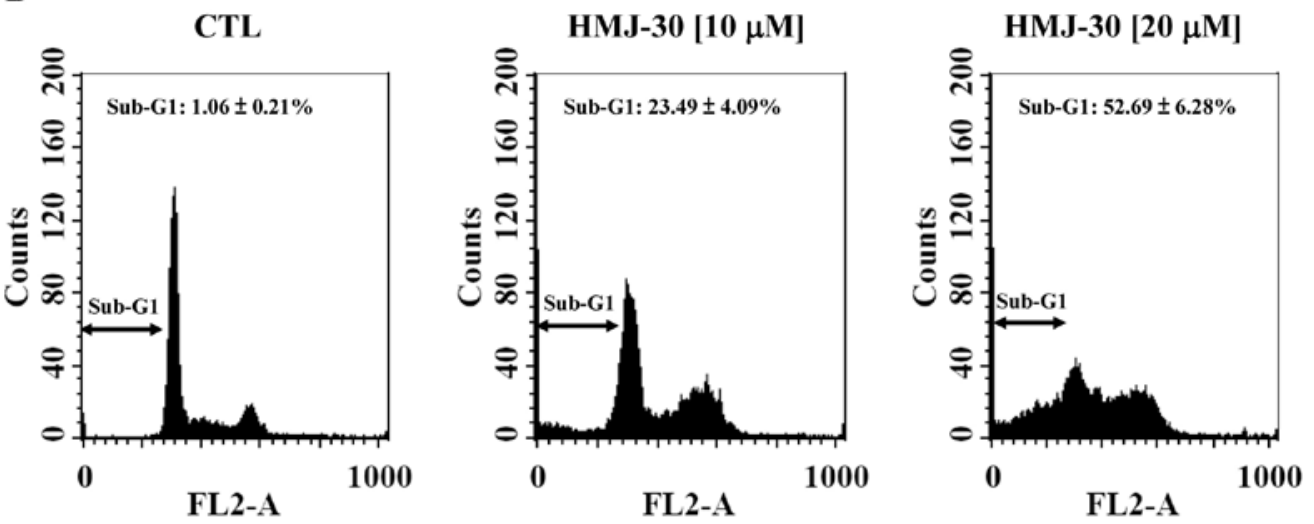

C

D
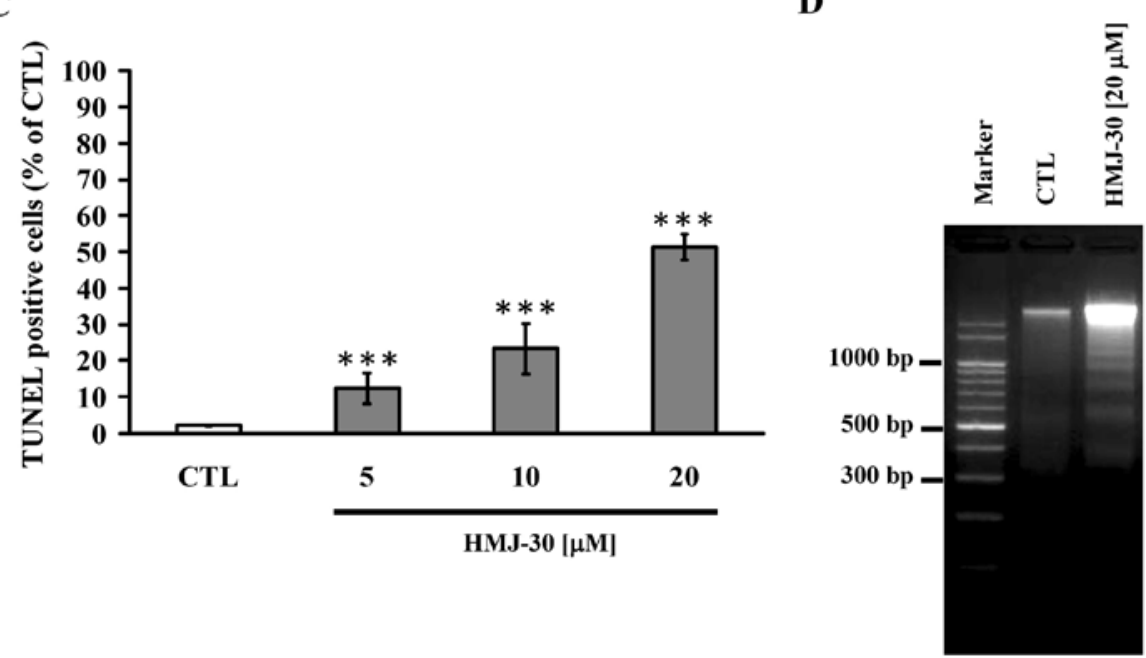

Figure 3. Cytotoxic effect of HMJ-30 and induction of apoptotic death in HUVECs. Cells were exposed to different concentrations of HMJ-30 (5, 10 or $20 \mu \mathrm{M}$ ) for $24 \mathrm{~h}$. (A) Cell morphological changes following HMJ-30 treatment were photographed under a phase-contrast microscope. (B) Profiles from flow cytometric analysis revealed the percentage of the sub-G1 population (apoptotic cells). (C) The quantitative value of TUNEL-positive cells was determined as the mean $\pm \operatorname{SEM}(\mathrm{n}=3) ;{ }^{* * *} \mathrm{p}<0.001$, significant difference compared with the untreated control (CTL). (D) Enrichment of DNA ladder was subjected to agarose gel electrophoresis as described in Materials and methods.

using the Student's t-test. $\mathrm{p}<0.001$ was considered to indicate a statistically significant result.

\section{Results}

HMJ-30 inhibits the angiogenesis of HUVECs in vitro. To evaluate the angiogenesis targeting potential of HMJ-30 in vitro, we carried out a series of angiogenic cellular functional assays in HUVECs. The viability of the HUVECs was significantly decreased in the HMJ-30-treated groups in a concentration- and time-dependent manner (Fig. 1B). Subsequently, we conducted a tube formation assay to determine the effect of HMJ-30 on tube-like network formation of HUVECs. Cells were placed on a Matrigel-coated plate with endothelial cell growth media with VEGF. HUVECs formed robust and elongated tube-like structures in the control group. In contrast, treatment with HMJ-30 concentration-dependently inhibited the formation of tube-like networks (Fig. 1C). Transwell migration assay was performed to evaluate the migratory behavior of HUVECs. The migration of endothelial cells is a pivotal step in the formation of new vessels. HMJ-30 significantly suppressed cell migration in a concentration-dependent manner (Fig. 1D). Overall, these results provide evidence that HMJ-30 inhibited the angiogenic activity of HUVECs in vitro.

HMJ-30 suppresses angiogenesis in vivo and ex vivo. Among various animal model systems established to investigate the mechanisms underlying angiogenesis, the chick embryo 

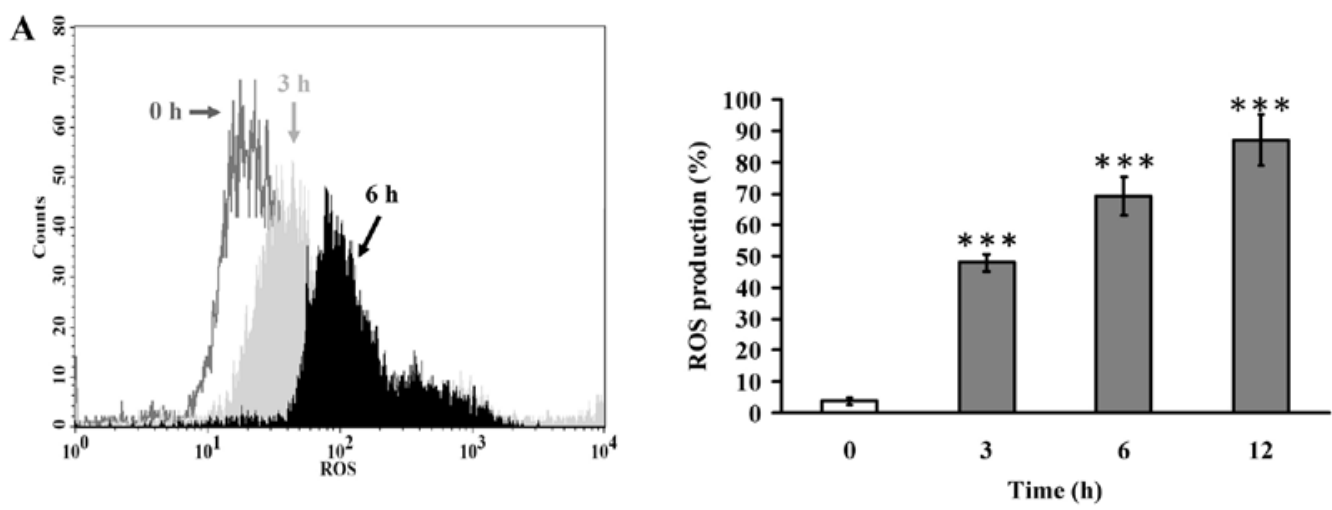

B

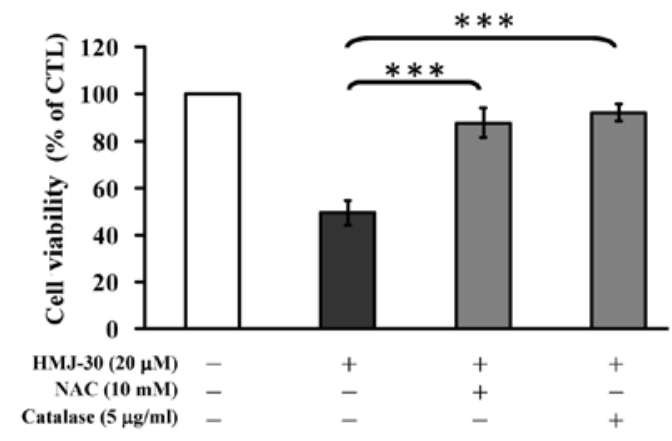

C

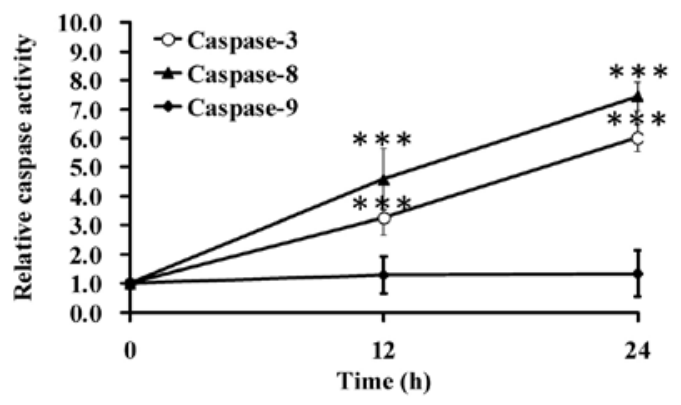

D

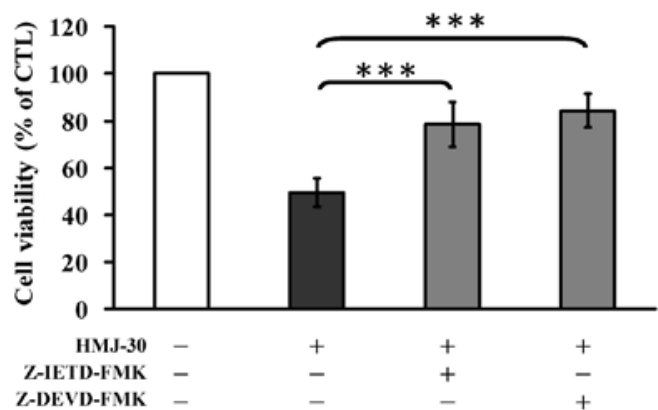

Figure 4. ROS production and levels of extrinsic caspases in HMJ-30-treated HUVECs. Cells were treated with $20 \mu \mathrm{M}$ HMJ-30 for the indicated time intervals. (A) ROS production was analyzed using $\mathrm{H}_{2}$ DCF-DA and by flow cytometry. The profile was quantified for DCF-positive cells utilizing BD CellQuest Pro software. (B) Cells were pretreated with NAC and catalase for $1 \mathrm{~h}$ before HMJ-38 treatment, and cell viability was determined by MTT assay. (C) The cell lysates were tested for caspase-3/-8/-9 activities as described in Materials and methods; (D) Cell viability was analyzed by MTT assay after cells were pretreated with or without Z-IETD-FMK and Z-DEVD-FMK followed by treatment with HMJ-30. All values are expressed as the mean \pm SEM $(n=3){ }^{* * *} p<0.001$, significant difference as compared with the untreated control or HMJ-30 treatment only cells. ROS, reactive oxygen species.

model and the mouse Matrigel plug have been well developed to analyze the anti-angiogenic or pro-angiogenic potential of compounds $(27,31)$. Both assays were conducted to verify the anti-angiogenic activity of HMJ-30 in vivo. The chorioallantoic membrane is the specialized and highly vascularized tissue of the avian embryo. The capillary plexus was dense and appeared as a honeycomb network in the control group. However, in the HMJ-30 treatment groups, vessel branching and sprouting were significantly reduced (Fig. 2A). Aortic ring vessel sprouting around the aortic rings stimulated by VEGF was observed in the untreated control, but this was markedly disrupted by HMJ-30 at 10 and $20 \mu \mathrm{M}$ to form a network of vessels (Fig. 2B). Angiogenesis in Matrigel plugs can be induced by VEGF in the mouse Matrigel plug assay. The plug in the control group contained abundant erythrocyte-filled vessels, indicating the formation of neovascularization, whereas only few vessels were observed in the HMJ-30-treated plugs (Fig. 2C). Additionally, we also measured the amount of hemoglobin contained in the plugs for quantification. The hemoglobin quantity was markedly reduced after treatment with HMJ-30 as compared to the control group (Fig. 2C). Taken together, these findings provide strong evidence of HMJ-30 to exhibit an anti-angiogenic response in vivo.

HMJ-30 induces apoptosis in HUVECs. We earlier confirmed that HMJ-30 inhibited cell proliferation of HUVECs in vitro. HUVECs detached from the surface of the plate and morphologic shrinkage was noted in the HMJ-30-treated cells, whereas cells of the control group were well spread with normal morphology (Fig. 3A). On the other hand, to determine the mechanism triggered by HMJ-30, we investigated the DNA content and apoptotic population by flow cytometry. 
A

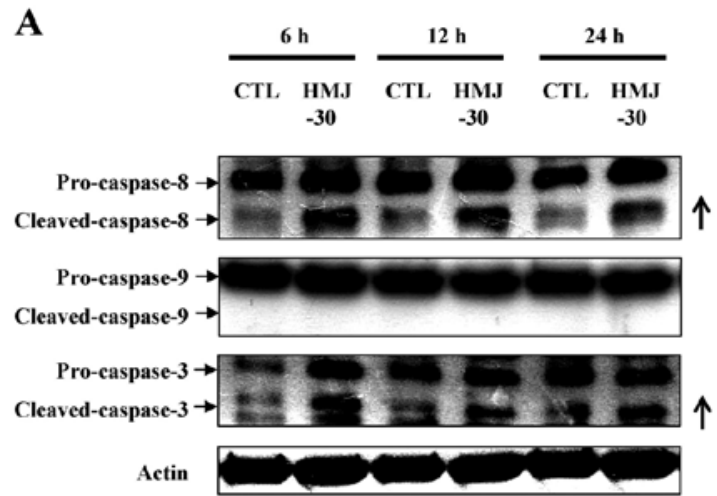

C

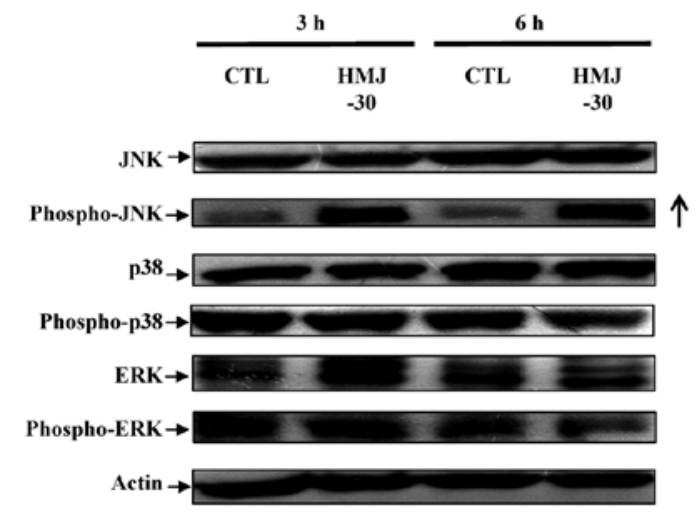

B
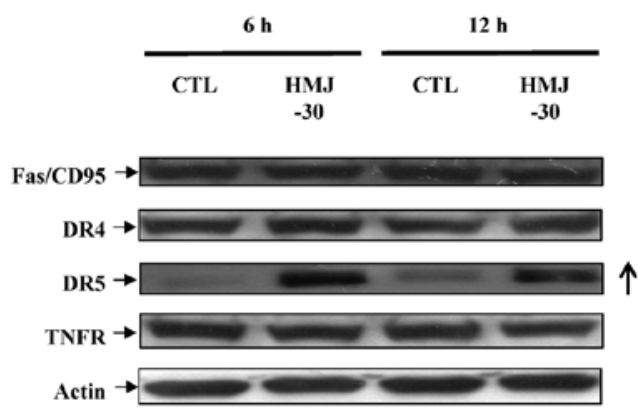

D

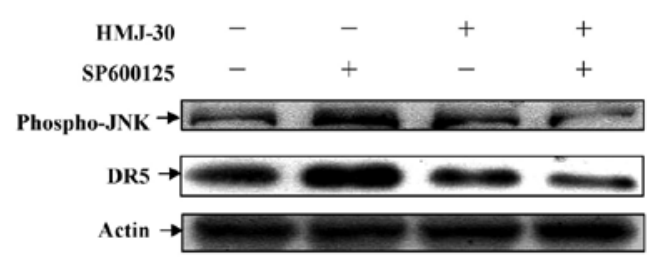

E

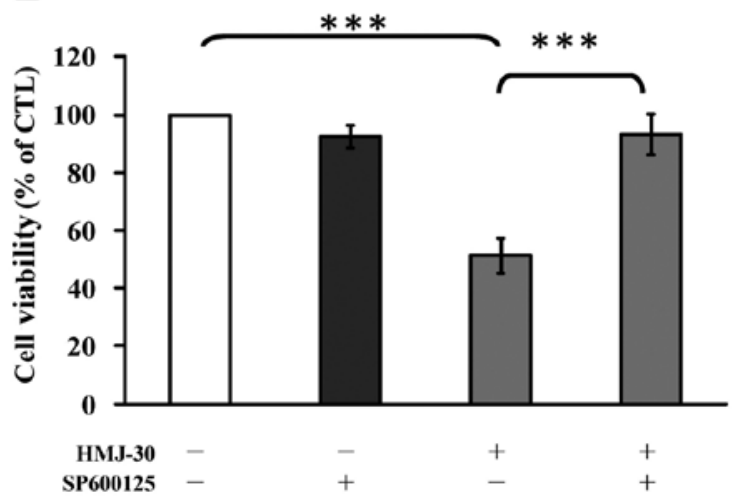

Figure 5. Effects of HMJ-30 on the expression of death receptors and MAPK JNK-related signaling in HUVECs. Cells were exposed to $20 \mu \mathrm{M}$ for the indicated periods of time and subjected to western blotting or MTT in vitro toxicology assay. Protein levels of (A) caspase-8, caspase-9 and caspase-3; (B) Fas/CD95, DR4, DR5 and TNFR; (C) JNK, phospho-JNK, p38, phospho-p38, ERK and phospho-ERK were shown. (D) HUVECs were pretreatment with SP600125 followed by HMJ-30 treatment, and phospho-JNK and DR5 expression was determined by immunoblot analysis. (E) Cell survival was assessed using MTT assay. Results are represented as the mean $\pm \operatorname{SEM}(n=3){ }^{* * * *} \mathrm{p}<0.001$, significant difference compared with the HMJ-30-treated group.

HMJ-30 induced an increase in hypodiploid (sub-G1 phase) cells (Fig. 3B). In addition, we conducted TUNEL assay and DNA agarose gel electrophoresis to evaluate DNA fragmentation. An increase in TUNEL-positive cells (Fig. 3C) and oligonucleosomal fragments (Fig. 3D) was observed in the HMJ-30-treated cells. Overall, these results demonstrated that HMJ-30 induced apoptotic cell death in the HUVECs.

HMJ-30 increases ROS generation in HUVECs. An increase in intracellular ROS has been demonstrated to play a critical role in eliciting an early response of apoptosis $(35,36)$. HMJ-30 time-dependently increased intracellular ROS levels (Fig. 4A). Furthermore, pretreatment with NAC or catalase significantly reduced HMJ-30-induced cell death (Fig. 4B). Based on these results, the induction of oxidative stress was required for HMJ-30-induced apoptosis in HUVECs.

HMJ-30 activates extrinsic apoptosis in HUVECs. To determine the molecular mechanism of apoptotic death induced by HMJ-30, we explored the activities of caspase-9, caspase-8, and caspase-3, respectively. The activities of caspase- 3 and caspase- 8 were significantly increased after treatment with HMJ-30 in a time-dependent manner (Fig. 4C). Moreover, pre-incubation with specific inhibitors of Z-IETD-FMK or Z-DEVD-FMK strongly increased cell viability compared with HMJ-30 treatment alone (Fig. 4D). However, caspase-9 activity was not significantly affected by HMJ-30 treatment (Fig. 4C). Overall, these data demonstrated that caspase-3/-8-dependent signaling plays a crucial role in HMJ-30-triggered apoptosis of HUVECs.

c-Jun N-terminal kinase (JNK)-mediated death receptor pathway in HUVECs is involved in HMJ-30-induced apoptosis. To elucidate the possible signaling pathway involved in HMJ-30-mediated apoptosis, the levels of associated proteins were evaluated. Treatment of HMJ-30 increased the cleaved forms of caspase- 3 and caspase- 8 rather than caspase- 9 (Fig. 5A), indicating activation of the extrinsic apoptotic pathway. DR5 (a death receptor-associated protein) was upregulated (Fig. 5B) and subsequently stimulated caspase- 8 expression to promote downstream effectors caspase- 3 to induce 


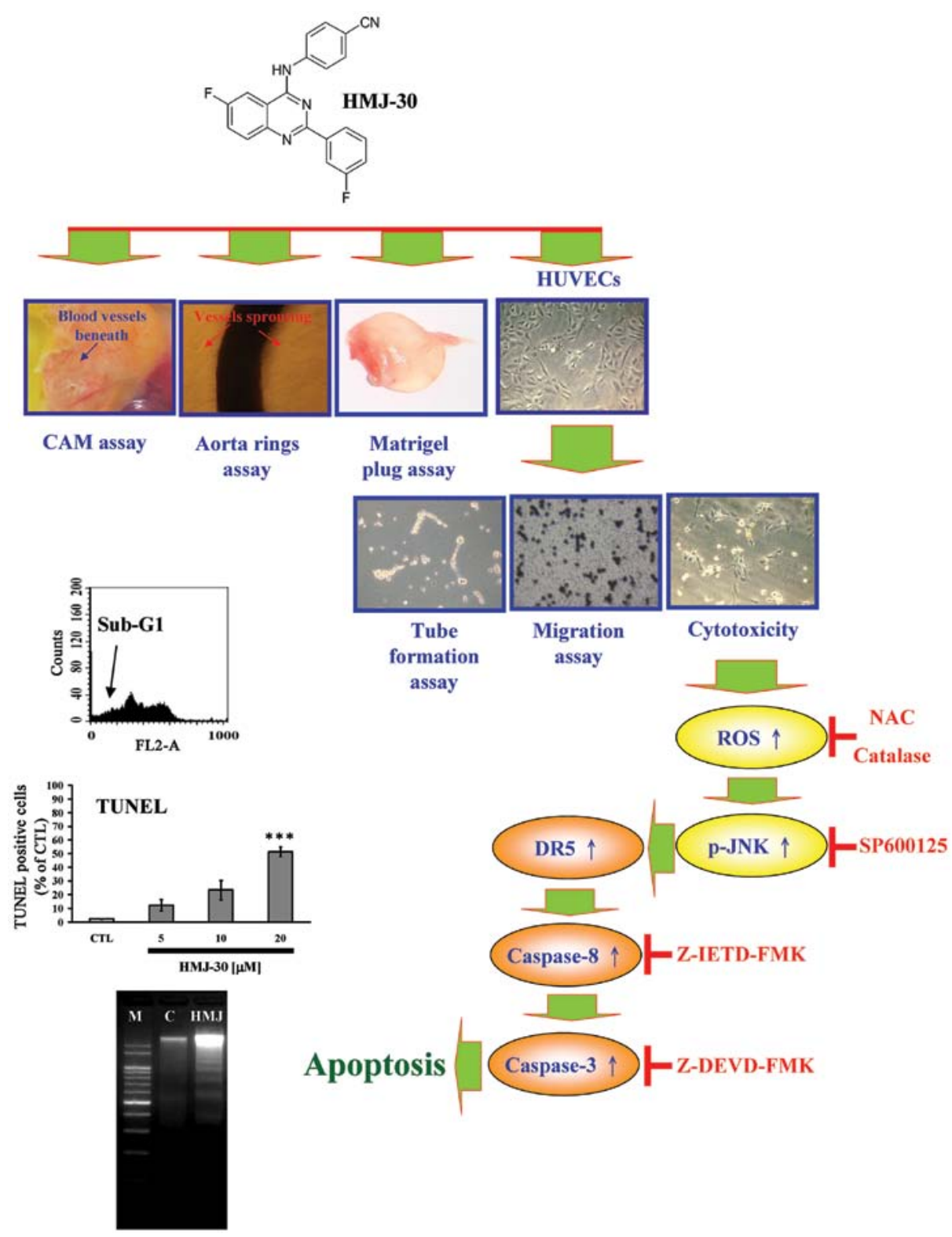

Figure 6. Schematic diagram of the molecular mechanism of HMJ-30. HMJ-30 regulates signaling pathways leading to anti-angiogenesis and apoptosis in HUVECs. HMJ-30, 6-fluoro-2-(3-fluorophenyl)-4-(cyanoanilino) quinazoline; HUVECs, human umbilical vein endothelial cells.

cell apoptosis (37). Additionally, mitogen-activated protein kinase (MAPK) pathway-associated proteins, including JNK, p38, extracellular signal-regulated kinase (ERK) and their phosphorylated molecules, were evaluated $(38,39)$. HMJ-30 increased the protein level of phosphorylated JNK (Fig. 5C), followed by an increase in DR5 dependent on the exposure time. Pretreatment with SP600125, a selective JNK inhibitor, effectively attenuated the phosphorylation of JNK and DR5 protein expression as well as markedly reversed the inhibition of cell viability in the HMJ-30-treated group. The results from our experimental approaches conclude that HMJ-30-induced apoptosis of HUVECs is mediated through DR5 and the JNK pathway.

\section{Discussion}

Blood vessels remove waste, deliver oxygen and nutrients to every part of the body; they also nourish cancer and provide pathways for malignant tumor cells to spread to other organs (1,3). Bevacizumab, a recombinant human monoclonal antibody against VEGF, was approved for clinical treatment to target the angiogenesis of cancer (9). However, approval of bevacizumab war questioned due to concerns about its toxicity and efficacy (15). Appearance of drug resistance ultimately results in the failure of VEGF-targeted therapies. HMJ-30 is able to prevent revascularization and disrupt existing vessels through induction of endothelial cell apoptosis. The different mechanism of this vascular targeting agent was therefore reported in this study.

Endothelial cells are able to disrupt the surrounding basement membrane to migrate toward angiogenic stimuli $(6,7,27)$. These cells subsequently form the necessary three-dimensional vessel structures to create new vessels through cell proliferation and reorganization $(6,27)$. The results of the tubeformation assay (Fig. 1C), migration assay (Fig. 1D), chick embryo assay (Fig. 2A), ex vivo rat aortic ring assay (Fig. 2B), 
and Matrigel plug assay (Fig. 2C) clearly indicated that HMJ-30 possessed strong anti-angiogenic activity in these in vitro, ex vivo and in vivo experiments.

Apoptosis is the process of programmed cell death, which plays a crucial role in diverse biological phenomenon and diseases $(7,44)$. Accumulating evidence indicates that endothelial cell apoptosis causes the disruption of blood vessel formation, resulting in the suppression of tumor progression $(7,43)$. Activation of the extrinsic initiator caspase- 8 with death effector domain would activate downstream of caspase-3, causing cell apoptosis (44). Our results showed that ROS generation played an important role in HMJ-30-induced apoptosis in HUVECs (Fig. 4A and B). Similar results were found by Chiu et al (22). HMJ-30 not only induced apoptotic death in HUVECs (Fig. 3), but also increased the activities and protein levels of caspase- 8 and caspase-3 in HMJ-30treated HUVECs. Furthermore, pre-incubation with their specific inhibitors restored the cell viability following HMJ-30 treatment. Thus, activation of the death receptor-associated caspase cascade was required for HMJ-30-induced apoptosis. Li et al (45) demonstrated that DR4 and DR5 proteins can be strongly expressed in HUVECs and human dermal microvessels. Incubation with tumor necrosis factor (TNF)-related apoptosis-inducing ligand (TRAIL) leads to caspase-8-dependent apoptosis death in HUVECs (45). The protein levels of TNF receptor superfamily members, including Fas, DR4, DR5 and TNFR, were therefore investigated in our study (Fig. 5B). Our data indicated that only DR5 was elevated following HMJ-30 treatment, suggesting that HMJ-30-induced apoptosis was mediated by the death-receptor pathway.

JNK signaling has been implicated in diverse cell physiological processes, including differentiation, proliferation and cellular stress-induced apoptosis. JNK signaling induces apoptosis by leading to secretion of death ligands to promote cytochrome $c$ release from mitochondria, or by phosphorylation of downstream pro-apoptotic proteins (46). Zhou et al (47) demonstrated that activation of JNK upregulates DR5 expression, which in turn leads to the activation of caspase8 -dependent apoptosis cascade in cancer cells. Our results showed that HMJ-30 increased the protein level of phosphoJNK, followed by an increase in DR5. Pretreatment with SP600125 effectively reversed the inhibition of cell viability (Fig. 5). These results indicated that HMJ-30-induced apoptosis and DR5 expression was mediated via a JNK-mediated mechanism. This is the first report to show the correlation between JNK signaling and DR5 in HUVECs.

The diverse pharmacological properties and anticancer activity of quinazoline have been previously reported $(18,19,21)$. The inhibition of tumor progression by quinazoline-like compounds is due to i) suppression of microtubule polymerization, ii) downregulation of the tyrosine kinase signaling pathway and iii) activation of apoptotic signaling cascades caused by intracellular stress $(24,26,33,36-38)$. In conclusion, we first demonstrated that HMJ-30 exhibits a potent anti-angiogenesis effect. The possible working mechanism of this agent might include i) prevention of new blood vessel formation, ii) elimination of existing vessels in tumors and iii) induction of apoptosis in HUVECs through upreregulation of DR5 and JNK-mediated extrinsic apoptosis signaling pathway (Fig. 6). These collective results provide pharma- cological basis for the therapeutic application of HMJ-30 in pathological angiogenesis-related diseases such as cancer.

\section{Acknowledgements}

The present study was supported in part by a research grant from the National Science Council of Taiwan, R.O.C. (NSC 102-2320-B-039-028-MY3 to T-S.W.).

\section{References}

1. Kerbel R and Folkman J: Clinical translation of angiogenesis inhibitors. Nat Rev Cancer 2: 727-739, 2002.

2. Tian T, Nan KJ, Wang SH, et al: PTEN regulates angiogenesis and VEGF expression through phosphatase-dependent and -independent mechanisms in HepG2 cells. Carcinogenesis 31: 1211-1219, 2010.

3. Hasan J, Shnyder SD, Bibby M, Double JA, Bicknel R and Jayson GC: Quantitative angiogenesis assays in vivo - a review. Angiogenesis 7: 1-16, 2004.

4. Stoletov K, Kato H, Zardouzian E, et al: Visualizing extravasation dynamics of metastatic tumor cells. J Cell Sci 123: 2332-2341, 2010.

5. Ferrara N: VEGF and the quest for tumour angiogenesis factors. Nat Rev Cancer 2: 795-803, 2002.

6. Xuan H,Zhao J, Miao J, Li Y, Chu Y and Hu F: Effect of Brazilian propolis on human umbilical vein endothelial cell apoptosis. Food Chem Toxicol 49: 78-85, 2011

7. Folkman J: Angiogenesis and apoptosis. Semin Cancer Biol 13: 159-167, 2003.

8. Plate KH, Breier G, Weich HA, Mennel HD and Risau W: Vascular endothelial growth factor and glioma angiogenesis: coordinate induction of VEGF receptors, distribution of VEGF protein and possible in vivo regulatory mechanisms. Int J Cancer 59: 520-529, 1994.

9. Strickler JH and Hurwitz HI: Bevacizumab-based therapies in the first-line treatment of metastatic colorectal cancer. Oncologist 17: 513-524, 2012

10. Sandler AB, Johnson DH and Herbst RS: Anti-vascular endothelial growth factor monoclonals in non-small cell lung cancer. Clin Cancer Res 10: 4258s-4262s, 2004

11. Yang JC, Haworth L, Sherry RM, et al: A randomized trial of bevacizumab, an anti-vascular endothelial growth factor antibody, for metastatic renal cancer. N Engl J Med 349: 427-434, 2003.

12. Ferrara N, Hillan KJ, Gerber HP and Novotny W: Discovery and development of bevacizumab, an anti-VEGF antibody for treating cancer. Nat Rev Drug Discov 3: 391-400, 2004.

13. Scott BJ, Quant EC, McNamara MB, Ryg PA, Batchelor TT and Wen PY: Bevacizumab salvage therapy following progression in high-grade glioma patients treated with VEGF receptor tyrosine kinase inhibitors. Neuro Oncol 12: 603-607, 2010.

14. Gild ML, Bullock M, Robinson BG and Clifton-Bligh R: Multikinase inhibitors: a new option for the treatment of thyroid cancer. Nat Rev Endocrinol 7: 617-624, 2011.

15. Bottsford-Miller JN, Coleman RL and Sood AK: Resistance and escape from antiangiogenesis therapy: clinical implications and future strategies. J Clin Oncol 30: 4026-4034, 2012.

16. Trowe T, Boukouvala S, Calkins K, et al: EXEL-7647 inhibits mutant forms of ErbB2 associated with lapatinib resistance and neoplastic transformation. Clin Cancer Res 14: 2465-2475, 2008.

17. Raghav N and Singh M: Design, synthesis and docking studies of bischalcones based quinazoline-2(1H)-ones and quinazoline-2 $(1 \mathrm{H})$-thiones derivatives as novel inhibitors of cathepsin B and cathepsin H. Eur J Pharm Sci 54: 28-39, 2014.

18. Bilbro J, Mart M and Kyprianou N: Therapeutic value of quinazoline-based compounds in prostate cancer. Anticancer Res 33: 4695-4700, 2013.

19. Xu L and Russu WA: Molecular docking and synthesis of novel quinazoline analogues as inhibitors of transcription factors $\mathrm{NF}-\kappa \mathrm{B}$ activation and their anti-cancer activities. Bioorg Med Chem 21: 540-546, 2013.

20. Chatterjee N, Das S, Bose D, et al: Exploring the anti-inflammatory activity of a novel 2-phenylquinazoline analog with protection against inflammatory injury. Toxicol Appl Pharmacol 264: 182-191, 2012.

21. Hour MJ, Huang LJ, Kuo SC, et al: 6-Alkylamino- and 2,3-dihydro-3'-methoxy-2-phenyl-4-quinazolinones and related compounds: their synthesis, cytotoxicity, and inhibition of tubulin polymerization. J Med Chem 43: 4479-4487, 2000. 
22. Chiu YJ, Hour MJ, Lu CC, et al: Novel quinazoline HMJ-30 induces U-2 OS human osteogenic sarcoma cell apoptosis through induction of oxidative stress and up-regulation of ATM/p53 signaling pathway. J Orthop Res 29: 1448-1456, 2011.

23. Ren T, Qing Y, Dai N, et al: Apurinic/apyrimidinic endonuclease 1 induced upregulation of fibroblast growth factor 2 and its receptor 3 induces angiogenesis in human osteosarcoma cells. Cancer Sci 105: 186-194, 2014.

24. Wu PP, Liu KC, Huang WW, et al: Triptolide induces apoptosis in human adrenal cancer NCI-H295 cells through a mitochondrial-dependent pathway. Oncol Rep 25: 551-557, 2011.

25. Yang JS, Hour MJ, Huang WW, Lin KL, Kuo SC and Chung JG: MJ-29 inhibits tubulin polymerization, induces mitotic arrest, and triggers apoptosis via cyclin-dependent kinase 1-mediated Bcl-2 phosphorylation in human leukemia U937 cells. J Pharmacol Exp Ther 334: 477-488, 2010.

26. Pan SL, Guh JH, Peng CY, et al: YC-1 [3-(5'-hydroxymethyl-2'-furyl)-1-benzyl indazole] inhibits endothelial cel functions induced by angiogenic factors in vitro and angiogenesis in vivo models. J Pharmacol Exp Ther 314: 35-42, 2005.

27. Chiang JH, Yang JS, Lu CC, et al: Newly synthesized quinazolinone HMJ-38 suppresses angiogenetic responses and triggers human umbilical vein endothelial cell apoptosis through p53-modulated Fas/death receptor signaling. Toxicol Appl Pharmacol 269: 150-162, 2013.

28. Kiriakidis S, Hogemeier O, Starcke S, et al: Novel tempeh (fermented soyabean) isoflavones inhibit in vivo angiogenesis in the chicken chorioallantoic membrane assay. Br J Nutr 93: 317-323, 2005

29. Pyun BJ, Choi S, Lee Y, et al: Capsiate, a nonpungent capsaicin-like compound, inhibits angiogenesis and vascular permeability via a direct inhibition of Src kinase activity. Cancer Res 68: 227-235, 2008.

30. Pang X, Yi Z, Zhang X, et al: Acetyl-11-keto-beta-boswellic acid inhibits prostate tumor growth by suppressing vascular endothelial growth factor receptor 2-mediated angiogenesis. Cancer Res 69: 5893-5900, 2009.

31. Pang X, Yi Z, Zhang J, et al: Celastrol suppresses angiogenesis-mediated tumor growth through inhibition of AKT/mammalian target of rapamycin pathway. Cancer Res 70 : 1951-1959, 2010.

32. Chung JG, Yang JS, Huang LJ, et al: Proteomic approach to studying the cytotoxicity of YC-1 on U937 leukemia cells and antileukemia activity in orthotopic model of leukemia mice. Proteomics 7: 3305-3317, 2007.

33. Huang WW, Chiu YJ, Fan MJ, et al: Kaempferol induced apoptosis via endoplasmic reticulum stress and mitochondria-dependent pathway in human osteosarcoma U-2 OS cells. Mol Nutr Food Res 54: 1585-1595, 2010.

34. Lu CC, Yang JS, Chiang JH, et al: Novel quinazolinone MJ-29 triggers endoplasmic reticulum stress and intrinsic apoptosis in murine leukemia WEHI-3 cells and inhibits leukemic mice. PLoS One 7: e36831, 2012.
35. Yodkeeree S, Sung B, Limtrakul P and Aggarwal BB: Zerumbone enhances TRAIL-induced apoptosis through the induction of death receptors in human colon cancer cells: Evidence for an essential role of reactive oxygen species. Cancer Res 69: 6581-6589, 2009.

36. Prasad S, Yadav VR, Ravindran J and Aggarwal BB: ROS and CHOP are critical for dibenzylideneacetone to sensitize tumor cells to TRAIL through induction of death receptors and downregulation of cell survival proteins. Cancer Res 71: 538-549, 2011.

37. Aggarwal BB, Bhardwaj U and Takada Y: Regulation of TRAIL-induced apoptosis by ectopic expression of antiapoptotic factors. Vitam Horm 67: 453-483, 2004.

38. Iwai A, Shiozaki T and Miyazaki T: Relevance of signaling molecules for apoptosis induction on influenza A virus replication. Biochem Biophys Res Commun 441: 531-537, 2013.

39. Kannaiyan R, Shanmugam MK and Sethi G: Molecular targets of celastrol derived from Thunder of God Vine: potential role in the treatment of inflammatory disorders and cancer. Cancer Lett 303: 9-20, 2011

40. Chiang JH, Yang JS, Lu CC, et al: Effect of DNA damage response by quinazolinone analogue HMJ-38 on human umbilical vein endothelial cells: Evidence for $\gamma \mathrm{H} 2 \mathrm{~A}$.X and DNA-PK-dependent pathway. Hum Exp Toxicol 33: 590-601, 2014.

41. Yang JS, Hour MJ, Kuo SC, Huang LJ and Lee MR: Selective induction of G2/M arrest and apoptosis in HL-60 by a potent anticancer agent, HMJ-38. Anticancer Res 24: 1769-1778, 2004.

42. Lu CC, Yang JS, Chiang JH, et al: Inhibition of invasion and migration by newly synthesized quinazolinone MJ-29 in human oral cancer CAL 27 cells through suppression of MMP-2/9 expression and combined down-regulation of MAPK and AKT signaling. Anticancer Res 32: 2895-2903, 2012.

43. Tozer GM, Kanthou C and Baguley BC: Disrupting tumour blood vessels. Nat Rev Cancer 5: 423-435, 2005.

44. Portt L, Norman G, Clapp C, Greenwood M and Greenwood MT: Anti-apoptosis and cell survival: a review. Biochim Biophys Acta 1813: 238-259, 2011.

45. Li JH, Kirkiles-Smith NC, McNiff JM and Pober JS: TRAIL induces apoptosis and inflammatory gene expression in human endothelial cells. J Immunol 171: 1526-1533, 2003.

46. Herr I and Debatin KM: Cellular stress response and apoptosis in cancer therapy. Blood 98: 2603-2614, 2001.

47. Zou W, Liu X, Yue P, et al: c-Jun NH2-terminal kinasemediated up-regulation of death receptor 5 contributes to induction of apoptosis by the novel synthetic triterpenoid methyl-2-cyano-3,12-dioxooleana-1, 9-dien-28-oate in human lung cancer cells. Cancer Res 64: 7570-7578, 2004. 